\title{
Photometric Behaviour of the EXors V1118 Orionis and V1143 Orionis
}

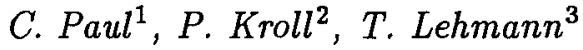 \\ 1 Astrophysikalisches Institut und Universitäts-Sternwarte Jena, Schillergäßchen 2, \\ 07740 Jena, Germany \\ 2 Sternwarte Sonneberg, 96515 Sonneberg, Germany \\ ${ }^{3}$ European Southern Observatory, Casilla 19001, Santiago 19, Chile
}

\begin{abstract}
We investigated the long-term photometrical behaviour of the EXors V1118 Ori and V1143 Ori on the basis of the Sonneberg Plate Archive. About 2800 plates were checked for outbursts of these stars. We found several dozens of plates where the stars appeared to be distinctly brighter than the plate limit. To get magnitudes we have scanned those plates and applied a special method of brightness determination which is currently used at Sonneberg Observatory. Because of the small number of plates we were able to extract only crude estimates of some typical time scales of outburst and quiescent phases.
\end{abstract}

\section{Introduction and brightness determinations}

EXors are a small group of very active $\mathrm{T}$ Tauri stars. Their main characteristics are strong outbursts of up to $5^{r m m}$, but the stars normally show only small amplitude irregular variations. The typical recurrence time scale of these events is of the order of a decade. FU Orionis stars (FUors) are another group of young low-mass stars with even stronger outbursts, which may last for a century.

Herbig (1977) pointed out that FU Orionis eruptions with strong intensities occur in T Tauri stars, while eruptions are absent on the main sequence, and there should exist similar objects with minor activity. EX Lupi was the first object and prototype (that's why they are called EXors).

Two recently found EXors are V1118 Ori (Chanal's object) and V1143 Ori (Sugano's object), which we studied on the basis of the Sonneberg plate archive.

For the derivation of magnitudes we have scanned the plates on which the stars are visible. V1143 Ori is an object without bright stars in its neighbourhood. Therefore the fitting method of Kroll \& Neugebauer (1993) was suitable to obtain reliable magnitudes. The accuracy $\sigma$ of these values is about 0.2 to 0.3 is mainly due to the rather poor plate quality (e.g., due to short exposure times). The star V1118 Ori could not be measured with the fitting method. It is embedded in an H II region and is located very near to the bright star V372 Ori. In this case the program fails, and the traditional method of Argelander was used, the stellar magnitude was estimated visually using a plate microscope. Since the comparison stars are also embedded in the H II region, the variable 
had to be linked to stars not only with similar magnitude but also with similar background level. We estimate the accuracy to be $\Delta \mathrm{m} \sim 0{ }^{\mathrm{m}} 5$.

\section{Results}

We examined about 2800 photographic patrol plates, taken between 1928 and 1993, for visibility of the investigated stars. In most cases, both stars were not visible because of the insufficient plate limit of $m_{\mathrm{pg}} \sim 12^{\mathrm{m}} 5 \ldots 14^{\mathrm{m}}$ of the shortfocus (patrol) cameras.

From the $\sim 50$ astrograph and Schmidt plates $\left(f \sim 160 \mathrm{~cm}, m_{\mathrm{pg} / \mathrm{lim}} \sim\right.$ $17^{\mathrm{m}} \ldots 18^{\mathrm{m}}$ ) on which V1143 Ori was found and from the patrol plates, it appeared that this star never flared brighter than $m_{\mathrm{pg}}=12.5$ during this time. The variable V1118 Ori, however, owing to the vicinity of the bright star V372 Ori, could not be found on patrol plates at all.

For V1118 Ori, discovered by Chanal in 1984, only a few pre-discovery observations were published (Gasparian et al. 1987). However, with the data of the Sonneberg plates we found that there were further flares in the past. Fig. 1 shows these few data. Chanal's object was visible at the following dates: 1939 Feb. 24 with $m_{\mathrm{pg}} \sim 14^{\mathrm{m}} 0,1956$ Feb. 5 with $m_{\mathrm{pg}} \sim 14^{\mathrm{m}} 0$ and 1961 Feb. 16 with $m_{\mathrm{pg}} \sim 14.0$. The other data confirm the outburst in 1982 , which probably had its maximum in 1983. Further data were not available. It is interesting that V1118 Ori flared on 1983 Dec. 5 to a photographic brightness of $m_{\mathrm{pg}} \sim 12.5$. Compared with the literature that seems to be the brightest value ever recorded (assuming $m_{\mathrm{pg}} \simeq m_{\mathrm{B}}$ ).

V1143 Ori showed also an outburst in 1982/83 and, after several brightenings, probably a second one in 1989 . This star was active in the past inb a similar way as V1118 Ori (Fig. 2).

Our data confirm the outburst in 1983. Further results are a brightening of about 2 mag (photographically) in 1939 (Feb. 22, < 17. ${ }^{\mathrm{m}} 7$; Feb. 23, 17. 1 ; Feb. $24,15.8$ ) and a possible outburst between $\sim 1966$ and $\sim 1970$ (1965 Oct. $22,<$ $16^{\mathrm{m}} 5 ; 1967$ Feb. $6,14^{\mathrm{m}} 2 ; 1969$ Oct. $6,14^{\mathrm{m}} 0 ; 1972$ Feb. $15,<14^{\mathrm{m}} 7$ - photographic magnitudes). This conclusion is in agreement with the lightcurve published by Gasparian et al., showing a possible post-outburst activity in September 1970. On the other hand, Chavira et al. (1985) claimed that V1118 Ori showed no outburst from 1944 to the beginning of 1982 .

\section{Discussion}

V1118 Ori and V1143 Ori exhibited outbursts or brightenings in the last few decades. However, owing to our sparse material it is not possible to derive rising times or duration of maxima.

On the other hand, according to the published data (mainly from the electronic AFOEV database) it is obvious that the timescales of these events reached several years at most. 


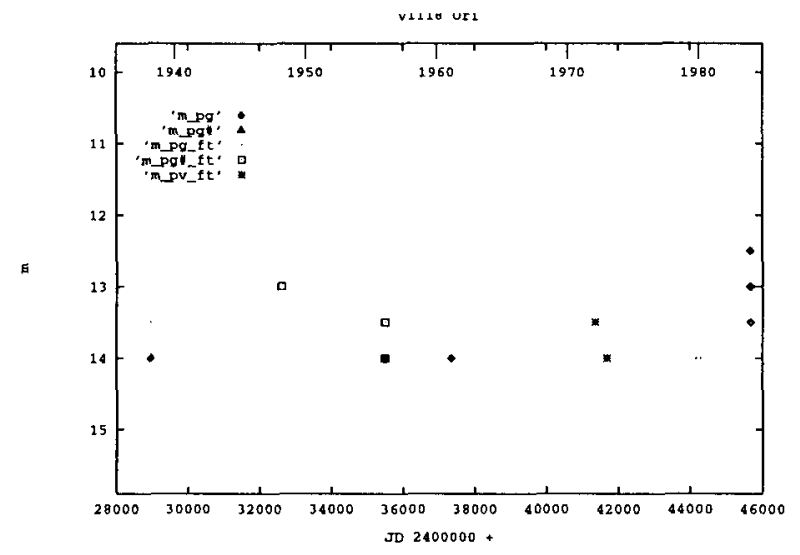

Fig. 1. Light-curve of V1118 Ori $\left(m_{\mathrm{pg}}-\right.$ photographic magnitudes, $m_{\mathrm{pg} \#}$ - uncertain photogr. mag., other - fainter than).

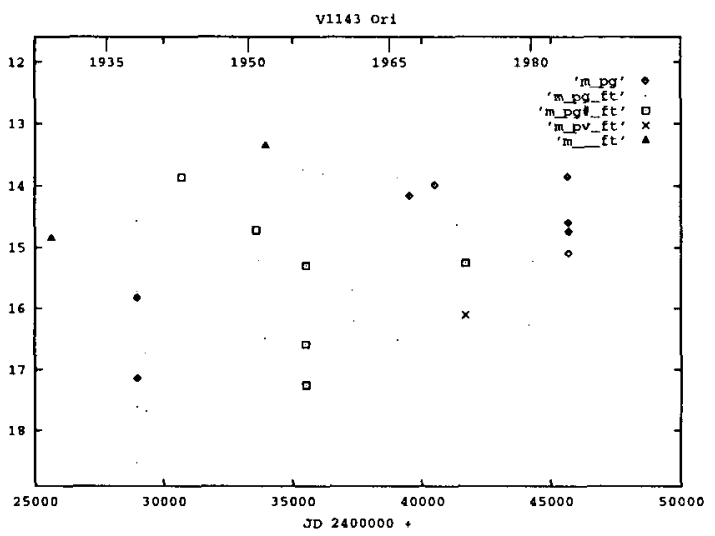

Fig. 2. Light-curve of V1143 Ori ( $m_{\mathrm{pg}}$ - photographic magnitudes, other - fainter than).

Acknowledgement: This work was supported by grant 2JN12A of Bundesministerium für Forschung und Technologie (Paul) und by grant 052 S0 524 of DARA/ DESY (Kroll).

\section{References}

Kroll P., Neugebauer G., 1993, A\&A 273, 341

Gasparian K.G., et al., 1987, IBVS 3024

Chavira E., et al., 1985, IBVS 2746

Herbig G.H., 1977, ApJ 217, 693 